\title{
Hemostasia compresiva con balón vs. inyección de epinefrina en sangrado inmediato postesfinterotomía
}

\author{
Lourdes G. Pedroza-Terán, Edgar G. Beltrán-Campos¹, Alejandra Arellano-Pérez', \\ Yolanda Zamorano-Orozco ${ }^{1}$ y Víctor H. Ahumada-Topete ${ }^{2}$ \\ ${ }^{1}$ Departamento de Endoscopia Gastrointestinal, Hospital Regional N. ${ }^{\circ}$ Carlos Mac Gregor Sánchez Navarro; ${ }^{2}$ Centro de Investigación en \\ Enfermedades Infecciosas, Instituto Nacional de Enfermedades Respiratorias. Ciudad de México, México
}

\begin{abstract}
Resumen
Introducción: El sangrado postesfinterotomía tiene una incidencia variable, desde un 0.3-2\% e incrementándose hasta un 9.6\% en algunos casos, y una mortalidad del $0.04 \%$. Aproximadamente el 10-30\% de las esfinterotomías pueden presentar hemorragia; el sangrado inmediato generalmente se autolimita, pero en ocasiones amerita realizar hemostasia intraprocedimiento y no se han estandarizado las diferentes opciones terapéuticas en estos casos. Objetivo: Evaluar la eficacia del uso de hemostasia por compresión con balón comparada con inyección de epinefrina en el sangrado inmediato postesfinterotomía. Material y métodos: Estudio retrospectivo, observacional y analítico. Se incluyeron pacientes con sangrado inmediato postesfinterotomía de enero de 2017 a marzo de 2020, se agruparon de acuerdo con la terapéutica utilizada, se evaluaron las características generales, factores de riesgo, hemostasia inicial y resangrado; se hizo regresión logística multivariante para identificar factores de riesgo de resangrado. Resultados: Un total de 60 pacientes en dos grupos: epinefrina 21 pacientes y balón 39 pacientes. La incidencia de sangrado inmediato fue del $8.21 \%$, la hemostasia inicial se logró en el 100\% de los casos. Predominó el sangrado leve en ambos grupos (14 [66.6\%] grupo epinefrina y 38 [97.4\%] grupo balón; $p=0.005$ ). Únicamente los pacientes con sangrado moderado presentaron resangrado: 7 (33.3\%) en el grupo epinefrina y $1(2.6 \%)$ en el grupo balón $(p=0.001)$. En el análisis multivariante el edema de papila tuvo mayor significancia (odds ratio: 6.5 ; intervalo de confianza del 95\%: 0.9-126; $p=0.05$ ). No se registraron efectos adversos, complicaciones o defunciones. Conclusiones: La hemostasia con balón es una herramienta útil, eficaz y segura, en algunos casos superior a epinefrina en el control del sangrado inmediato postesfinterotomía.
\end{abstract}

Palabras clave: Hemorragia. Esfinterotomía. Epinefrina. Oclusión con balón.

\section{Hemostasis with balloon occlusion versus epinephrine injection in immediate post-sphincterotomy bleeding}

\section{Abstract}

Background: The incidence of post-sphincterotomy bleeding is variable, from $0.3-2 \%$, increasing to $9.6 \%$ in some cases; with a mortality of $0.04 \%$. Approximately $10-30 \%$ of sphincterotomies may present hemorrhage, immediate bleeding is generally self-limited, however, sometimes intra-procedure hemostasis is warranted; The different therapeutic options in these cases have not been standardized. Objective: To evaluate the efficacy of the use of balloon compression hemostasis compared with epinephrine injection in immediate post-sphincterotomy bleeding. Material and methods: Retrospective, obser-

Correspondencia:

*Lourdes G. Pedroza-Terán

E-mail: drapedroza.lourdes@gmail.com
Disponible en internet: 28-06-2021 Endoscopia. 2021;32(3):75-80 www.endoscopia-ameg.com
Fecha de aceptación: 14-04-2021 DOI: 10.24875/END.20000069 
vational and analytical study. All patients who presented immediate post-sphincterotomy bleeding from January 2017 to March 2020 were included and grouped according to the therapy used, the general characteristics, risk factors, initial hemostasis and rebleeding were evaluated; multivariate logistic regression was performed to identify risk factors for rebleeding. Results: $A$ total of 60 patients divided into two groups: adrenaline $=21$ patients and balloon $=39$ patients. The incidence of immediate bleeding was $8.21 \%$ and initial hemostasis was achieved in $100 \%$ of the cases. Mild bleeding predominated in both groups (14 $(66.6 \%)$ adrenaline group and $38(97.4 \%)$ balloon group; $p=0.005)$. Only patients with moderate bleeding had rebleeding (7 (33.3\%) adrenaline group and $1(2.6 \%)$ balloon group, $p=0.001)$. In the multivariate analysis, ampullary edema had greater significance OR $6.5(0.9-12695 \% \mathrm{Cl}, p=0.05)$. No adverse effects, complications or deaths were recorded. Conclusions: Balloon hemostasis is a useful, effective and safe tool, in some cases superior to epinephrine in the control of immediate post-sphincterotomy bleeding.

Key words: Hemorrhage. Sphincterotomy. Epinephrine. Balloon occlusion.

\section{Introducción}

El sangrado postesfinterotomía es el segundo evento adverso más común relacionado con la realización de colangiopancreatografía retrógrada endoscópica (CPRE). Su incidencia es variable, desde el $0.3-2 \%$, incrementándose hasta el $9.6 \%$ en algunos casos; la mortalidad es del $0.04 \%$. Aproximadamente el $10-30 \%$ de las esfinterotomías pueden presentar sangrado, que generalmente es leve y autolimitado ${ }^{1,2}$.

Los principales factores de riesgo de sangrado son: coagulopatía (odds ratio [OR]: $3.32 ; p \leq 0.001$ ), colangitis (OR: $2.59 ; p \leq 0.001$ ), terapia anticoagulante (OR: 5.11; $p \leq 0.001$ ), inexperiencia del endoscopista (OR: $2.17 ; p \leq 0.002$ ), sangrado durante procedimiento (OR: $1.74 ; p<0.004)^{2}$, cifra de plaquetas $<50,000 \mathrm{~mm}^{3}$ (OR: 35.30; $p=0.002$ ), cirrosis (OR: $2.05 ; p<0.0001$ ), enfermedad renal crónica en hemodiálisis (OR: 13.30; $p<0.001$ ), así como el divertículo periampular, esfinterotomía previa y litiasis biliar impactada ${ }^{3-5}$. La tasa de resangrado varía del 5 al $22 \%$; algunos predictores son la cifra de bilirrubinas $>10 \mathrm{mg} / \mathrm{dl}(\mathrm{OR}: 3.55 ; \mathrm{p}=0.008$ ) y el sangrado severo (OR: 48.74; $p<0.001)^{6}$.

Las guías clínicas recomiendan la administración local de epinefrina como terapia de primera línea, la cual se ha estudiado principalmente en el sangrado tardío ${ }^{1,2}$, ya sea sola o combinada con terapia térmica, la tasa de éxito es del 92 vs. $100 \%$ respectivamente $(p=0.44)$, con una recurrencia del 16 vs. $12.1 \%$, respectivamente ${ }^{7-9}$. Existen otras terapias alternativas, como: terapia térmica, colocación de hemoclip, prótesis metálica totalmente cubierta autoexpandible, polvos hemostáticos, embolización guiada por ultrasonido endoscópico y/o aplicación de coils, con resultados satisfactorios ${ }^{10-14}$. Para el control del sangrado en casos refractarios se recomienda la embolización por angiografía o cirugía ${ }^{15,16}$.

El taponamiento con balón extractor de litos o balón dilatador en el sangrado intraprocedimiento se ha descrito como una opción para tener un control temporal y adecuada visualización del sitio de sangrado ${ }^{17,18}$. Es una herramienta frecuentemente utilizada en nuestro medio para control del sangrado inmediato, sin embargo, no hay estudios clínicos que evalúen esta terapia.

El objetivo principal del estudio fue comparar el uso de hemostasia por compresión con balón contra la inyección de epinefrina 1:10,000 en el sangrado inmediato postesfinterotomía. Además de conocer los factores de riesgo, recurrencia y factores de riesgo de resangrado, así como definir los eventos adversos.

\section{Métodos}

Estudio retrospectivo, observacional y analítico. Se recabaron todos los estudios de CPRE realizados de enero de 2017 a marzo de 2020 en el Servicio de Endoscopia del Hospital Regional Carlos Mac Gregor Sánchez Navarro, del Instituto Mexicano del Seguro Social. Se seleccionaron todos los pacientes que presentaron sangrado inmediato postesfinterotomía y se formaron dos grupos de acuerdo con la terapéutica utilizada: grupo epinefrina y grupo balón (Fig. 1). Los estudios de laboratorio y datos demográficos se obtuvieron de expedientes clínicos, sistema electrónico y solicitudes con las que fueron referidos.

Se realizó comparación de medias y desviación estándar con prueba de $t$ de Student para variables paramétricas y prueba de $\chi^{2}$ para variables no paramétricas; se establecieron promedios para variables continuas; se identificaron factores de riesgo de resangrado mediante análisis de regresión logística multivariante. Se consideró el valor de $p \leq 0.05$ como estadísticamente significativo. El software utilizado fue SPSS versión 25.0.

El sangrado inmediato postesfinterotomía se definió como aquel que se presentó durante o inmediatamente después del procedimiento y continuó 2-3 min posterior 


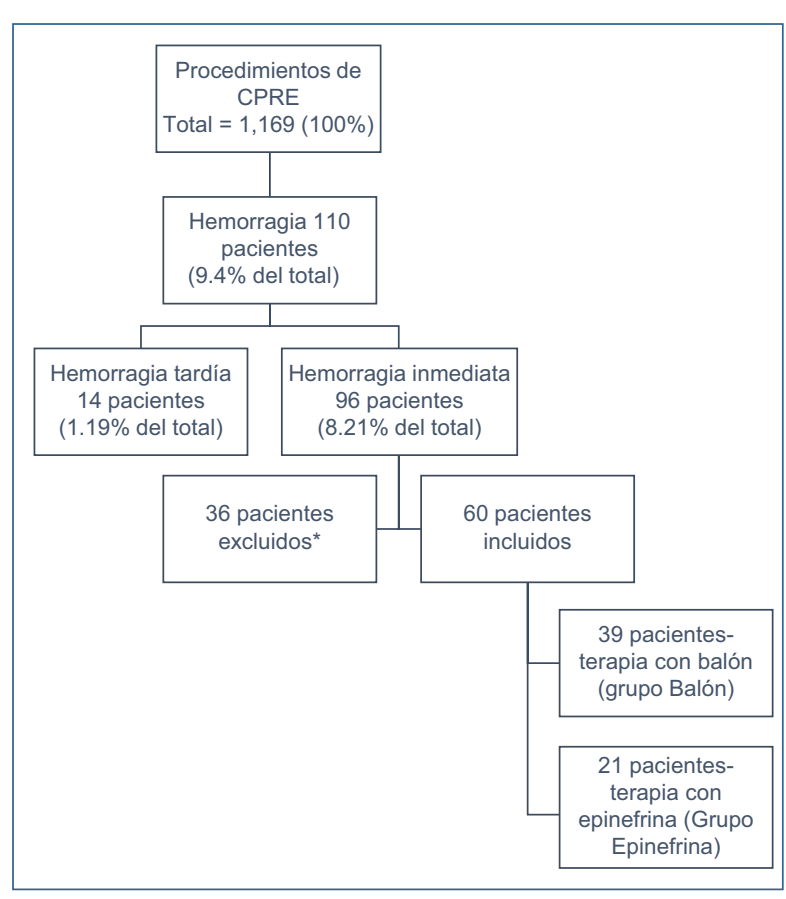

Figura 1. Diagrama de flujo de la selección de pacientes en el periodo de tiempo estudiado.

* Los pacientes excluidos recibieron una terapia endoscópica diferente.

CPRE: colangiopancreatografía retrógrada endoscópica.

a la esfinterotomía. La gravedad de la hemorragia se clasificó en: leve, moderada o grave de acuerdo con la clasificación de Cotton, et al. ${ }^{1,2}$. El estigma de sangrado se clasificó en capa/chorro, descrito por el médico que realizó el estudio. La presencia de coagulopatía se definió como tiempo de protombina $>3 \mathrm{~s}$ respecto al control o índice internacional normalizado $>2.5 \mathrm{y} / \mathrm{o}$ trombocitopenia a la cifra de plaquetas $<50,000 / \mu \mathrm{l}$.

Se incluyeron pacientes mayores de 18 años, de ambos sexos, a quienes se les realizó CPRE con esfinterotomía o precorte y presentaron sangrado inmediato, requiriendo hemostasia endoscópica intraprocedimiento mediante inyección de epinefrina o compresión con balón extractor de litos; ningún paciente se encontraba con uso de anticoagulantes al momento del estudio. Se excluyeron pacientes con sangrado tardío, si recibieron otra terapia o si tenían datos incompletos.

\section{Procedimiento}

Los estudios fueron realizados con los equipos del servicio: duodenoscopio Fujifilm Ed-530x o Pentax Ed3490tk; ERBE VIO 300s, modo ENDOCUT I. Esfinterotomo triple lumen Wilson Cook/Boston Scientific.
Papilotomo de aguja/precorte (needle/knife) Wilson Cook/Boston. Técnica hemostasia con inyección de epinefrina 1;10,000: inyección perilesional con inyector 7 fr/aguja $23 \mathrm{Ga}$, dosis variable entre 2-10 ml. Técnica hemostasia con balón: compresión de la papila con balón extractor de litos sobre el sitio donde se realizó esfinterotomía/precorte, tamaño promedio del balón 12$15 \mathrm{~mm}$, durante 1-2 min, máximo 5 minutos hasta lograr hemostasia. La elección del tratamiento fue de acuerdo con el criterio del médico que realizó el estudio.

\section{Resultados}

Se realizaron 1,169 (100\%) estudios de CPRE de enero de 2017 a marzo de 2020 en el Hospital Regional Carlos Mac Gregor Sánchez Navarro, de los cuales $110(9.4 \%)$ presentaron hemorragia relacionada con el procedimiento, solo 96 (8.21\%) pacientes tuvieron sangrado inmediato postesfinterotomía, de los cuales se incluyeron 60 pacientes, que se dividieron en dos grupos de acuerdo con la terapia utilizada: 21 pacientes recibieron terapia con epinefrina (grupo epinefrina) y 39 pacientes hemostasia con balón (grupo balón) (Fig. 1).

La edad promedio fue de $61.1( \pm 20)$ años en el grupo epinefrina y $58.5( \pm 20)$ años en el grupo balón ( $p=$ 0.63 ); predominó el sexo femenino en ambos grupos, sin diferencias entre grupos $(p=0.33)$. El diagnóstico más frecuente fue coledocolitiasis: $12(57.1 \%)$ en el grupo epinefrina y $19(48.7 \%)$ en el grupo balón. Seguido de colangitis: $2(9.5 \%)$ en el grupo epinefrina y $7(17.9 \%)$ en el grupo balón. El tercer diagnóstico más frecuente fue fuga biliar, $3(14.3 \%)$ en el grupo epinefrina, y síndrome ictérico, 7 (17.9\%) en el grupo balón ( $p$ $=0.39$ ).

Los factores de riesgo fueron: coagulopatía 1 (2.6\%) y cirrosis $1(2.6 \%)$ en el grupo balón ( $p=0.45$ ); divertículo periampular $2(9.5 \%)$ en el grupo epinefrina y $4(10.3 \%)$ en el grupo balón ( $p=0.9)$; colangitis $2(9.5 \%)$ y $8(20.5 \%)$ en los grupos epinefrina y balón, respectivamente $(p=0.27)$; edema de papila $8(38.1 \%)$ en el grupo epinefrina y $18(46.2 \%)$ en el grupo balón ( $p=$ 0.49); esfinterotomía 16 (76.2\%) en el grupo epinefrina y $36(92.3 \%)$ en el grupo balón ( $p=0.08)$; precorte 7 (33.3\%) y 6 (15.4\%) en los grupos epinefrina y balón, respectivamente $(p=0.10)$. Como complicaciones: un paciente de cada grupo presentó pancreatitis postCPRE $(p=0.65)$ y un paciente ameritó hemotransfusión por resangrado en el grupo epinefrina $(p=0.16)$. En el grupo epinefrina, $13(61 \%)$ de los estudios fueron realizados por residentes. así como 13 (33.3\%) del 
Tabla 1. Características clínicas, endoscópicas y factores de riesgo en ambos grupos

\begin{tabular}{|c|c|c|c|}
\hline \multicolumn{4}{|c|}{ Características clínicas, endoscópicas y factores de riesgo en ambos grupos (media $\pm \mathrm{DE}$ ), n (\%) } \\
\hline Variable & Epinefrina ( $\mathbf{n}=\mathbf{2 1}$ ) & Balón (n = 39) & p \\
\hline Edad (DE) & $61.1( \pm 20)$ años & $58.5( \pm 20)$ años & 0.63 \\
\hline $\begin{array}{l}\text { Sexo }(\%) \\
\text { Femenino } \\
\text { Masculino }\end{array}$ & $\begin{array}{l}13(61.9 \%) \\
8(38.1 \%)\end{array}$ & $\begin{array}{l}20(51.3 \%) \\
19(48.7 \%)\end{array}$ & 0.33 \\
\hline $\begin{array}{l}\text { Diagnósticos }(\%) \\
\text { Coledocolitiasis } \\
\text { Colangitis } \\
\text { Probable ampuloma } \\
\text { Quiste de colédoco } \\
\text { Fuga biliar } \\
\text { Síndrome ictérico en estudio } \\
\text { Pancreatitis biliar }\end{array}$ & $\begin{array}{c}12(57.1 \%) \\
2(9.5 \%) \\
1(4.8 \%) \\
1(4.8 \%) \\
3(14.3 \%) \\
1(4.8 \%) \\
1(4.8 \%)\end{array}$ & $\begin{array}{c}19(48.7 \%) \\
7(17.9 \%) \\
1(2.6 \%) \\
0 \\
2(5.2 \%) \\
7(17.9 \%) \\
3(7.7 \%)\end{array}$ & 0.39 \\
\hline $\begin{array}{l}\text { Factores de riesgo (\%) } \\
\text { Coagulopatía } \\
\text { Cirrosis } \\
\text { Divertículo periampular } \\
\text { Colangitis } \\
\text { Edema de papila } \\
\text { Esfinterotomía } \\
\text { Precorte }\end{array}$ & $\begin{array}{c}0 \\
0 \\
2(9.5 \%) \\
2(9.5 \%) \\
8(38.1 \%) \\
16(76.2 \%) \\
7(33.3 \%)\end{array}$ & $\begin{array}{c}1(2.6 \%) \\
1(2.6 \%) \\
4(10.3 \%) \\
8(20.5 \%) \\
18(46.2 \%) \\
36(92.3 \%) \\
6(15.4 \%)\end{array}$ & $\begin{array}{l}0.45 \\
0.45 \\
0.90 \\
0.27 \\
0.49 \\
0.08 \\
0.10\end{array}$ \\
\hline $\begin{array}{l}\text { Tamaño esfinterotomía (mm) } \\
<5 \mathrm{~mm} \\
5-10 \mathrm{~mm} \\
>10 \mathrm{~mm}\end{array}$ & $\begin{array}{c}1(4.7 \%) \\
14(66.6 \%) \\
0\end{array}$ & $\begin{array}{c}3(7.6 \%) \\
28(71.7 \%) \\
5(12.8 \%)\end{array}$ & 0.31 \\
\hline $\begin{array}{l}\text { Estudios de laboratorio (DE) } \\
\text { Hb basal } \\
\text { Bilirrubinas totales } \\
\text { Fosfatasa alcalina } \\
\text { Hb postsangrado }\end{array}$ & $\begin{array}{c}13.1 \mathrm{~g} / \mathrm{dl}( \pm 1.9) \\
5.4 \mathrm{mg} / \mathrm{dl}( \pm 4.2) \\
368 \mathrm{Ul} /( \pm 376) \\
11 \mathrm{~g} / \mathrm{dl}( \pm 2.9)\end{array}$ & $\begin{array}{l}13.5 \mathrm{~g} / \mathrm{dl}( \pm 1.7) \\
7.7 \mathrm{mg} / \mathrm{dl}( \pm 4.2) \\
308 \mathrm{Ul} / \mathrm{l}( \pm 230) \\
12.4 \mathrm{~g} / \mathrm{dl}( \pm 1.6)\end{array}$ & $\begin{array}{l}0.45 \\
0.24 \\
0.39 \\
0.35\end{array}$ \\
\hline Transfusión sanguínea (\%) & $1(4.8 \%)$ & 0 & 0.16 \\
\hline Pancreatitis post-CPRE (\%) & $1(4.8 \%)$ & $1(2.6 \%)$ & 0.65 \\
\hline $\begin{array}{l}\text { Médico que realizó el estudio (\%) } \\
\text { Endoscopista experimentado } \\
\text { Endoscopista en adiestramiento }\end{array}$ & $\begin{array}{c}8(39.0 \%) \\
13(61.0 \%)\end{array}$ & $\begin{array}{l}26(66.7 \%) \\
13(33.3 \%)\end{array}$ & 0.03 \\
\hline
\end{tabular}

DE: desviación estándar; Hb: hemoglobina; CPRE: colangiopancreatografía retrógrada endoscópica.

grupo balón ( $p=0.03$ ). El resto de las variables evaluadas no mostraron diferencias entre grupos (Tabla 1).

En todos los pacientes se logró hemostasia inicial $(100 \%)$. El sangrado en capa fue el más común, $17(80.9 \%)$ en el grupo epinefrina y $39(100 \%)$ en el grupo balón, con sangrado a chorro solo en el grupo de epinefrina $(4[19 \%])(p=0.005)$.

De acuerdo con la gravedad de la hemorragia, $14(66.6 \%)$ casos del grupo epinefrina y $38(94.7 \%)$ del grupo balón fueron sangrados leves; 7 (33.3\%) en el grupo epinefrina y $1(2.6 \%)$ en el grupo balón tuvieron sangrado moderado $(\mathrm{p}=0.001)$; todos los pacientes con sangrado moderado presentaron resangrado $(p=0.001)$ (Tabla 2).

La recurrencia del sangrado en la reintervención endoscópica se manifestó como sangrado en capa en todos los casos, solo 2 (28.6\%) pacientes del grupo epinefrina y $1(100 \%)$ paciente del grupo balón tuvieron sangrado inactivo, el resto requirió tratamiento endoscópico, que fue seleccionado de acuerdo con el criterio del endoscopista (Tabla 3).

En el análisis multivariante para factores asociados a recurrencia, el que mostró mayor significancia fue el edema de papila (OR: 10.9; intervalo de confianza del 95\%: 0.9-126; $p=0.05$ ); el resto de variables 
Tabla 2. Estigmas, gravedad y desenlace de la hemorragia en la intervención inicial

\begin{tabular}{|l|c|c|c|}
\hline \multicolumn{3}{|c|}{ Estigmas, gravedad y desenlace de la hemorragia en la } \\
intervención inicial
\end{tabular}

analizadas, sin resultados significativos (Tabla 4). No se registraron efectos adversos ni muertes.

\section{Discusión}

Este es el primer estudio que compara la hemostasia con balón vs. epinefrina en el sangrado inmediato postesfinterotomía. La incidencia máxima en el mundo es de hasta el $9.6 \% \%^{1,2}$, en nuestro estudio se reportó en un $9.4 \%$ y en sangrado inmediato de un $8.21 \%$, lo que está relacionado probablemente con que somos un hospital escuela.

Freeman, et al. ${ }^{2}$ fueron los primeros en definir los factores de riesgo en el sangrado postesfinterotomía, posteriormente se encontraron otros factores y también para riesgo de recurrencia ${ }^{4-6}$. En nuestro estudio, aunque algunos pacientes tenían factores de riesgo conocidos, la mayoría no mostró relevancia estadística, probablemente relacionado con el tamaño de muestra. Como hallazgo, un porcentaje importante de pacientes tenía edema de papila, el cual en el análisis multivariante fue la variable con mayor significancia como factor de recurrencia, probablemente esto se deba a un incremento en la vascularidad por inflamación, sin embargo, no existe literatura al respecto, ni otros estudios, por lo que sería un área de oportunidad para estudios prospectivos, controlados, para definir si puede considerarse como tal.

Se realizaron más esfinterotomías que precortes (en nuestro centro describimos la longitud en milímetros), ninguno tuvo relación con recurrencia; cabe mencionar que al paciente con coagulopatía se le realizó precorte por canulación difícil, posterior a canulación presentó sangrado y se optó por hemostasia con balón.

Similar al estudio de Tsuo, et al. ${ }^{8}$, nuestra tasa de éxito inicial en ambas terapias fue del $100 \%$, pero nuestra recurrencia fue mayor, la cual correspondió a pacientes con sangrado moderado principalmente, y correlaciona con otros estudios donde a mayor gravedad, mayor riesgo de recurrencia ${ }^{6}$. La mayoría de los procedimientos en el grupo epinefrina fueron realizados por médicos residentes (aunque siempre son supervisados y asistidos de ser necesario por un endoscopista experto), fue el grupo con mayor incidencia de sangrado a chorro, sangrado moderado y resangrado, pero con menor número de pacientes; sabemos que la inexperiencia es un factor conocido como riesgo de hemorragia, inicialmente mostró diferencia significativa vs. el grupo balón, pero en el análisis multivariante no logró asociarse con recurrencia.

La clasificación del estigma de sangrado es una clasificación subjetiva, no está validada como tal, sin embargo, puede orientar al endoscopista a preferir un método terapéutico sobre otro, como en nuestro estudio, donde para el control del sangrado a chorro se prefirió el uso de esta terapia. No se reportaron efectos adversos, ni mortalidad; en los dos pacientes que presentaron pancreatitis fueron leves, con una incidencia dentro de lo esperado y no se relacionaron con la terapéutica utilizada.

Nuestro estudio tiene algunas debilidades, al ser retrospectivo y con grupos ligeramente heterogéneos; sin embargo, se lograron los objetivos. Podemos concluir que la hemostasia con balón es una herramienta útil y segura, en algunos casos superior a epinefrina, se encuentra accesible en la mayoría de las unidades de endoscopia y podría considerarse como una opción terapéutica para el control del sangrado inmediato postesfinterotomía, sin embargo, se requieren estudios prospectivos para estandarizar el uso de esta terapia; así como también para confirmar si el edema de papila es un factor de riesgo de resangrado.

\section{Financiamiento}

La presente investigación no ha recibido ninguna beca específica de agencias de los sectores públicos, comercial o sin ánimo de lucro.

\section{Conflicto de intereses}

Los autores declaran no tener conflicto de intereses alguno. 
Tabla 3. Características iniciales de los pacientes con resangrado y reintervención endoscópica, n (\%)

\begin{tabular}{|c|c|c|c|c|}
\hline \multicolumn{2}{|c|}{ Pacientes con resangrado } & $\begin{array}{c}\text { Epinefrina } \\
\qquad(\mathrm{n}=7)\end{array}$ & $\begin{array}{l}\text { Balón } \\
(n=1)\end{array}$ & $\mathbf{p}$ \\
\hline \multicolumn{2}{|c|}{$\begin{array}{l}\text { Características del sangrado inicial } \\
\text { Sangrado moderado } \\
\text { Sangrado a chorro } \\
\text { Sangrado en capa }\end{array}$} & $\begin{array}{l}7(100 \%) \\
2(28.5 \%) \\
5(71.4 \%)\end{array}$ & $\begin{array}{c}1(100 \%) \\
0 \\
1(100 \%)\end{array}$ & $\begin{array}{l}0.001 \\
0.005\end{array}$ \\
\hline \multicolumn{2}{|c|}{ Hallazgos endoscópicos (reintervención) } & & & \\
\hline Tipo sangrado & Terapia endoscópica & & & \\
\hline $\begin{array}{l}\text { Sangrado en capa } \\
\text { Sangrado en capa } \\
\text { Sangrado en capa } \\
\text { Sangrado en capa } \\
\text { Sangrado inactivo }\end{array}$ & $\begin{array}{l}\text { Hemostasia con balón } \\
\text { Epinefrina + Prótesis plástica 10x10Fr } \\
\text { Electrocoagulación (precorte) } \\
\text { Epinefrina +electrocoaculación (precorte) } \\
\text { No }\end{array}$ & $\begin{array}{l}1(14.2 \%) \\
1(14.2 \%) \\
1(14.2 \%) \\
2(28.6 \%) \\
2(28.6 \%)\end{array}$ & $1(100 \%)$ & \\
\hline
\end{tabular}

Tabla 4. Análisis multivariante para predictores de resangrado

\section{Análisis multivariante para predictores de resangrado}

\begin{tabular}{|l|c|c|c|}
\hline Predictor & OR & IC 95\% & p \\
\hline Divertículo duodenal & 0.5 & $0.01-19.3$ & 0.71 \\
\hline Edema de papila & 10.9 & $0.9-126.2$ & 0.05 \\
\hline Coledocolitiasis & 3.1 & $0.45-22.4$ & 0.24 \\
\hline Esfinterotomía & 7.3 & $0.34-159.5$ & 0.50 \\
\hline Precorte & 1.5 & $0.074-31.04$ & 0.78 \\
\hline Bilirrubinas > 10 mg/dl & 0.9 & $0.89-1.03$ & 0.16 \\
\hline Endoscopista en adiestramiento & 4.9 & $0.44-55$ & 0.19 \\
\hline
\end{tabular}

OR: odds ratio; IC: intervalo de confianza.

\section{Responsabilidades éticas}

Protección de personas y animales. Los autores declaran que para esta investigación no se han realizado experimentos en seres humanos ni en animales.

Confidencialidad de los datos. Los autores declaran que han seguido los protocolos de su centro de trabajo sobre la publicación de datos de pacientes.

Derecho a la privacidad y consentimiento informado. Los autores declaran que en este artículo no aparecen datos de pacientes.

\section{Bibliografía}

1. Dumonceau JM, Kapral C, Aabakken L, Papanikolaou IS, Tringali A, Vanbiervliet G, et al. ERCP-related adverse events: European Society of Gastrointestinal Endoscopy (ESGE) Guideline. Endoscopy. 2020:52(2):127-49.

2. Chandrasekhara V, Khashab M, Muthusamy VR, Acosta RD, Agrawal D, Bruining DH, et al. Adverse events associated with ERCP. Gastrointest Endosc. 2017;85(1):32-47.
3. Mashiana HS, Dhaliwal AS, Sayles H, Dhindsa B, Yoo JW, Wu Q. Endoscopic retrograde cholangiopancreatography in cirrhosis - a systematic review and meta-analysis focused on adverse events. World J Gastrointest Endosc. 2018;10(11):354-66.

4. Nakaji S, Hirata N, Matsui H, Shiratori T, Kobayashi M, Yoshimura S. Hemodialysis is a strong risk factor for post-endoscopic sphincterotomy bleeding in patients with choledocholithiasis. Endosc Int Open. 2018;6(5):E568-E574.

5. Tsai MC, Wang CC, Wang YT, Yang TW, Chen HY, Tseng MH, et al. Major bleeding risk of endoscopic sphincterotomy versus endoscopic papillary balloon dilatation in hemodialysis patients. Saudi J Gastroenterol. 2019;25(2):106-12.

6. Lee MH, Tsou YK, Lin CH, Lee CS, Liu NJ, Sung KF, et al. Predictors of re-bleeding after endoscopic hemostasis for delayed post-endoscopic sphincterotomy bleeding. World J Gastroenterol. 2016;22(11):3196201.

7. Tsou YK, Lin CH, Liu NJ, Tang JH, Sung KF, Cheng CL, et al. Treating delayed endoscopic sphincterotomy-induced bleeding: Epinephrine injection with or without thermotherapy. World J Gastroenterol. 2009; 15(38):4823-8.

8. Wilcox CM, Canakis J, Mönkemüller KE, Bondora AW, Geels W. Patterns of bleeding after endoscopic sphincterotomy, the subsequent risk of bleeding, and the role of epinephrine injection. Am J Gastroenterol. 2004;99(2):244-8.

9. Chon HK, Kim TH. Endoclip therapy of post-sphincterotomy bleeding using a transparent cap-fitted forward-viewing gastroscope. Surg Endosc. 2017;31(7):2783-8.

10. Liu F, Wang GY, Li ZS. Cap-assisted hemoclip application with forwardviewing endoscope for hemorrhage induced by endoscopic sphincterotomy: a prospective case series study. BMC Gastroenterol. 2015; $15: 135$.

11. Aranez JL, Miller J, Hughes M, DeCross A, Kaul V. A novel, duodenoscope-friendly endoscopic clip for treating massive upper-Gl bleeding secondary to a Dieulafoy lesion. VideoGIE. 2018;3(7):205-6.

12. Katsinelos P, Kountouras J, Chatzimavroudis G, Zavos C, Fasoulas K, Katsinelos T, et al. Endoscopic hemostasis using monopolar coagulation for postendoscopic sphincterotomy bleeding refractory to injection treatment. Surg Laparosc Endosc Percutan Tech. 2010;20(2):84-8.

13. Guzmán-Calderón E, Ruiz F, Casellas JA, Martinez-Sempere J, Medina-Prado L, Aparicio JR. Endoscopic ultrasound-guided injection of coils for the treatment of refractory post-ERCP bleeding. Endoscopy. 2020:52(8):702-3

14. Rustagi T, Jamidar PA. Endoscopic retrograde cholangiopancreatography-related adverse events: general overview. Gastrointest Endosc Clin N Am. 2015;25(1):97-106.

15. So YH, Choi YH, Chung JW, Jae HJ, Song SY, Park JH. Selective embolization for post-endoscopic sphincterotomy bleeding: technical aspects and clinical efficacy. Korean J Radiol. 2012;13(1):73-81.

16. Dunne R, McCarthy E, Joyce E, McEniff N, Guiney M, Ryan JM, et al. Post-endoscopic biliary sphincterotomy bleeding: an interventional radiology approach. Acta Radiol. 2013;54(10):1159-64.

17. Bae SS, Lee DW, Han J, Kim HG. Risk factor of bleeding after endoscopic sphincterotomy in average risk patients. Surg Endosc. 2019;33(10):3334-40.

18. Kahaleh M, Freeman M. Prevention and management of post-endoscopic retrograde cholangiopancreatography complications. Clin Endosc. 2012;45(3):305-12. 